\title{
Analytical model and molecular dynamics simulations of the size dependence of flow stress in amorphous intermetallic nanowires at temperatures near the glass transition
}

\author{
Yujie Wei, ${ }^{1,2, *}$ Allan F. Bower, ${ }^{1, \dagger}$ and Huajian Gao ${ }^{1, \$}$ \\ ${ }^{1}$ Division of Engineering, Brown University, Box D, Providence, Rhode Island 02912, USA \\ ${ }^{2}$ Department of Mechanical Engineering, University of Alabama, P.O. Box 870276, Tuscaloosa, Alabama 35487, USA
}

(Received 10 September 2009; revised manuscript received 28 December 2009; published 2 March 2010)

\begin{abstract}
Molecular dynamics (MD) simulations are used to compute the flow stress of amorphous metallic nanowires that are deformed at temperatures near the glass transition. The simulations predict a strong size dependence of flow stress and predict the existence of a critical wire radius that minimizes the flow stress. Examination of the cross sections of the wires shows evidence of significant free-volume nucleation and diffusion during straining. The MD results are interpreted using a simple analytical model that assumes that the wire deforms by a combination of viscous flow, together with a diffusional deformation process in which free volume is continuously nucleated in the interior of the wire, and subsequently diffuses to the surface. The predictions of the model are in good qualitative agreement with simulation results. The analytical model is used to estimate the critical wire dimensions where this diffusional mechanism is likely to replace viscous creep at time scales that are inaccessible using MD simulations.
\end{abstract}

DOI: 10.1103/PhysRevB.81.125402

PACS number(s): 62.23.Hj, 62.20.F-, 66.30.Pa, 66.30.hh

\section{INTRODUCTION}

Amorphous materials have a wide range of attractive properties, including good structural stability and formability, and their unique microstructure and composition provides opportunities to tune their performance through microalloying. There is currently particular interest in developing techniques to fabricate and test submicron and nanoscale components of amorphous materials. ${ }^{1-3}$ This is partly motivated by applications: for example, metallic glasses have been successfully utilized for precision components such as miniaturized geared motors in microelectromechanical systems. ${ }^{4}$ In addition, tensile and compression tests on amorphous nanowires (NWs) provide a novel approach to probe the underlying mechanisms of deformation in metallic glasses, which have been the subject of much debate. Recent experimental observations of large tensile deformability ${ }^{1}$ and homogeneous compressive deformation ${ }^{2,3}$ in metallic glass nanopillars are particularly intriguing and suggest that the large surface area to volume ratio in these specimens provides a mechanism to prevent the formation of shear bands, which typically limit the ductility of bulk tensile specimens at room temperatures. Further, machine stiffness and sample size in combination may play a role for plasticity in metallic glass micropillars. ${ }^{5}$

Existing studies of the size dependence of flow stress in nanowires have largely focused on deformation at room temperatures. In this paper, our goal is to investigate flow mechanisms in nanowires at temperatures close to the glass transition. To this end, molecular dynamics (MD) simulations are used to model the uniaxial tensile deformation of amorphous $\mathrm{Cu}_{50} \mathrm{Zr}_{50}$ nanowires. Atomic interactions are approximated using a mixed embedded atom potential, which yields an onset glass transition temperature for the bulk amorphous glass of about $700 \mathrm{~K}$. Model nanowires with radii ranging from 3 to $20 \mathrm{~nm}$ are deformed at temperatures ranging from 300-1000 $\mathrm{K}$ and nominal strain rates of order $10^{8} \mathrm{~s}^{-1}$. The nanowires show no signs of developing the shear bands that typically limit the ductility of bulk amorphous specimens and instead fail by necking at strains that exceed $100 \%$. The flow stress of the wires is strongly sensitive to strain rate and temperature, suggesting that deformation occurs at least in part due to viscous flow. In addition, the flow stress of the nanowires (at fixed strain rate) is found to be strongly dependent on wire radius. The flow stress is minimized at a critical wire radius, which appears to be only weakly dependent on strain rate and temperature.

An examination of the cross sections of the nanowires shows evidence of extensive free-volume nucleation, as well as migration of atoms from the surface of the wire toward the interior. This suggests that, as the wire radius is reduced, the deformation mode transitions from predominantly viscous flow (in large wires) to a process that involves continuous nucleation of free volume within the bulk of the wire, which subsequently diffuses to the surface. Nucleation of free volume is known to play a central role in controlling deformation in amorphous materials. In particular, local free-volume nucleation and diffusion accommodates shearing in sheartransformation zones (STZs). ${ }^{6}$ It is thought that large gradients in free volume, such as those that occur in shear bands, can also give rise to diffusion. Long-range diffusion of free volume is not normally regarded as a mechanism of deformation, although fast diffusion of single elements in amorphous materials has been observed at sufficiently high temperatures. $^{7-10}$ Recent molecular dynamics simulations also suggest that long-range diffusion is feasible under certain conditions. ${ }^{11-13}$ Drawing an analogy from polycrystalline materials-where deformation is accommodated by competing mechanisms with relative contributions determined by different rates-we suggest that such kinetics may apply to the deformation in amorphous solids at sufficiently high temperatures.

To this end, we develop an approximate analytical model of deformation in amorphous nanowires by a combination of diffusion and viscous flow. The model gives closed-form expressions for the stress and strain rate distributions in the 


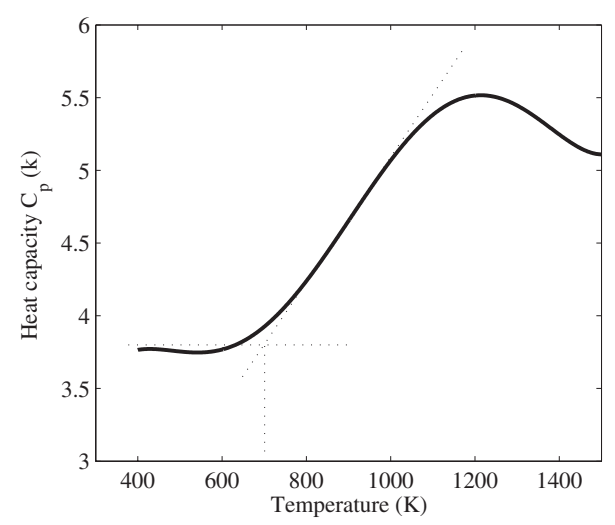

FIG. 1. Specific heat vs temperature for the binary metallic glass system $\mathrm{Cu}_{50} \mathrm{Zr}_{50}$ using a mixed EAM potential. A rapid increasing of the heat capacity (in units of $\mathrm{k}$, the Boltzmann constant) is observed for temperatures between 700 and $1100 \mathrm{~K}$ and the onset of glass transition temperature $T_{g}$ is determined to be around $710 \mathrm{~K}$.

wire, as well as the flow stress as a function of wire radius. With relevant model parameters estimated from MD simulations, the model predictions show good agreement with the simulation results.

Due to the limitations of molecular dynamics, direct numerical simulations are possible only for wires with very small diameters that are subjected to high strain rates. These conditions would be difficult to achieve in experiments. Consequently, we use our analytical model to estimate the conditions where the diffusional mode of deformation is likely to occur in preference to viscous creep in larger wires and lower strain rates. Our estimates suggest that, in $\mathrm{Zr}_{41.2} \mathrm{Ti}_{13.8} \mathrm{Cu}_{12.5} \mathrm{Ni}_{10.0} \mathrm{Be}_{22.5}$ (Vitreloy 1) deformed at $400 \mathrm{~K}$, diffusional flow may play a role in nanowires up to $100 \mathrm{~nm}$.

The remainder of this paper is organized as follows. In Sec. II, we describe some details and principal results of our MD simulations. In Sec. III, we present an analytical model of deformation by coupled viscous flow and diffusion and discuss briefly the behavior predicted by this model. The MD simulations are compared to the analytical model in Sec. IV and in Sec. V, we use the analytical calculations to estimate conditions under which diffusion is likely to replace viscous creep.

\section{MOLECULAR DYNAMICS SIMULATIONS}

Molecular dynamics simulations were performed on model nanowires of undercooled amorphous $\mathrm{Cu}_{50} \mathrm{Zr}_{50}$. This material has been extensively studied both experimentally ${ }^{14,15}$ and numerically. ${ }^{16-18}$ The system was idealized using the mixed embedded atom method (EAM) potential ${ }^{19}$ supplied in LAMMPS..$^{20}$ The glass transition temperature was estimated by computing the specific-heat capacity of the solid as a function of temperature using a $12 \mathrm{~nm}$ cubic unit cell consisting of 108000 atoms with periodic boundary conditions. These simulations were performed at constant temperatures and null pressure. Figure 1 shows the specific heat vs. temperature for the binary metallic glass system. A rapid change of heat capacity-which is associated with the glass transition-is observed at $710 \mathrm{~K}$. The estimates of glass transition temperature from our simulation agree reasonably well with experimental results $[650-730 \mathrm{~K}$ (Ref. 15)] and with MD simulations using different atomic interactions [e.g., $620 \mathrm{~K}$ (Ref. 18)].

Our simulation sample is prepared in following steps. Starting from a randomly mixed sample of $850000 \mathrm{Cu}$ atoms and $850000 \mathrm{Zr}$ atoms at $T=3000 \mathrm{~K}$ in a cuboid of 20 $\times 20 \times 70 \mathrm{~nm}^{3}$, we relax the system under periodic boundary condition and null pressure for $1 \mathrm{~ns}$. We then cool down the cuboid to $300 \mathrm{~K}$ at a rate of $-1000 \mathrm{~K} / \mathrm{ns}$. It is noted that the cooling rate also affects the properties of an amorphous sample in MD simulations. ${ }^{21}$ We note in passing that MD simulations will never be completely realistic; even if we anneal for the longest possible time, we will probably not achieve the true material state. The purpose of simulations shown here is to predict qualitative trends and to reveal the underlying physics.

Amorphous $\mathrm{Cu}_{50} \mathrm{Zr}_{50}$ nanowires were generated from a dog-bone-shaped cylindrical domain out of the cuboid, as shown in Fig. 2(a). A typical wire has diameter between 3 and $20 \mathrm{~nm}$, length ranging from 17 to $70 \mathrm{~nm}$, and contains between 8491 and 1055423 atoms. The radius of the wire is reduced slightly away from the two ends to minimize boundary effects. The length of the region with uniform radius is 3 times the wire diameter in all simulations. The sample was heated up to a designated temperature and then relaxed for $100 \mathrm{ps}$ at null pressure and constant temperature. The sample was deformed by fixing the atoms at one end and applying a constant velocity $v$ to the atoms at the other end of the specimen. The average of the virial stress component along the wire axis in the region with uniform radius is taken as the macroscopic stress of the wire during its deformation. At finite strains, this stress measure differs from either engineering stress or true stress, but can be interpreted as true stress for small deformations (while the wire deforms homogeneously).

Figure 2(c) shows the deformation of the $15 \mathrm{~nm}$ nanowire during straining at $300 \mathrm{~K}$ at a strain rate of $2 \times 10^{9} \mathrm{~s}^{-1}$. During the initial stages, the wire deforms homogeneously. A neck begins to form near the center of the specimen at a nominal strain of order $10 \%$ and the specimen eventually fails by necking. There is no evidence of shear band formation, which typically limits the ductility of bulk amorphous materials deformed at room temperature. Figure 2(d) shows the deformation at $1000 \mathrm{~K}$ and strain rate $2 \times 10^{9} \mathrm{~s}^{-1}$ : under these conditions, the wire deforms homogeneously up to $100 \%$ strain.

The corresponding stress-v-strain curves for the $15 \mathrm{~nm}$ wire are illustrated in Fig. 3 for temperatures ranging from 300 to $1000 \mathrm{~K}$ and strain rates of $2 \times 10^{8}$ and $2 \times 10^{9} \mathrm{~s}^{-1}$. The stress-strain behavior is clearly sensitive to both temperature and strain rate. Even at room temperature, a strain rate sensitivity of $m \approx 0.1$ is seen, where the stress exponent $m=\partial \ln \sigma / \partial \ln \dot{\varepsilon}$ can be interpreted as the slope of a graph of $\log (\sigma)$ as a function of $\log (\dot{\varepsilon})$. At $T=1000 \mathrm{~K}, m$ approaches 0.35 . The increase in rate sensitivity at high temperatures delays the development of a neck and at $1000 \mathrm{~K}$, the specimen deforms homogeneously up to $100 \%$ strain [see Fig. 2(d)]. 


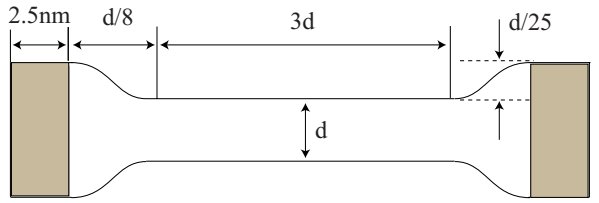

(a)

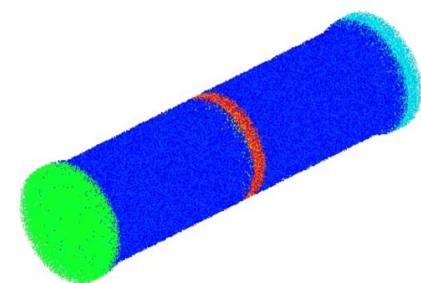

(b)

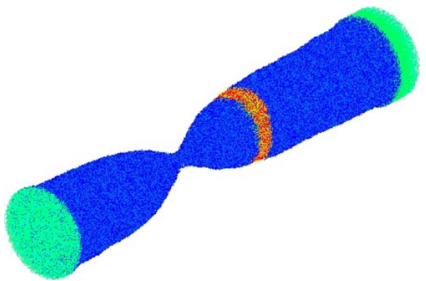

(c)

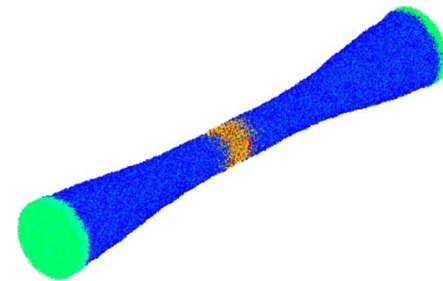

(d)

FIG. 2. (Color online) Typical glassy $\mathrm{Cu}_{50} \mathrm{Zr}_{50}$ nanowire studied in molecular dynamics simulations. Colored atoms at the two ends of the specimen are subjected to prescribed velocities to simulate uniaxial tensile straining at constant strain rates. Colored atoms near the center of the specimen are used to analyze deformation. (a) Layout of self-similar samples with diameter $d$. (b) Initial specimen. (c) Necking at 300 $\mathrm{K}$ and $2 \times 10^{9} \mathrm{~s}^{-1}$. (d) Homogeneous deformation at $1000 \mathrm{~K}$ and $2 \times 10^{9} \mathrm{~s}^{-1}$.

The influence of wire diameter on flow stress is illustrated in Fig. 4, which shows stress-v-nominal strain curves for nanowires with diameters between 3 and $20 \mathrm{~nm}$, deformed at $700 \mathrm{~K}$ (just below the glass transition temperature for the solid), and at strain rates of $2 \times 10^{9} \mathrm{~s}^{-1}$ (Fig. 4, left) and 2 $\times 10^{8} \mathrm{~s}^{-1}$ (Fig. 4, right). A size effect is predicted: wires with $3 \mathrm{~nm}$ diameter show higher strength than 5 and $8 \mathrm{~nm}$ wires and this behavior is consistent with recent simulations of room-temperature straining of $\mathrm{Ni}_{50} \mathrm{Zr}_{50}$ reported by Delogou, ${ }^{22}$ which showed an increase in strength as wire radius was reduced from 6 to $1 \mathrm{~nm}$. More interestingly, the strength of wires becomes higher as wire radius change from 8 to 10,15 , and $20 \mathrm{~nm}$.

We propose that this size effect during elevated temperature straining can be attributed to a change in the deformation mechanism as the wire diameter is reduced. Large nanowires deform predominantly by viscous flow (the deformation mechanism in bulk amorphous materials near the glass transition). In contrast, smaller nanowires appear to deform by a diffusional mechanism. In this process, free volume is continuously nucleated near the center of the wire and subsequently diffuses to the wire surface. Nucleation of free

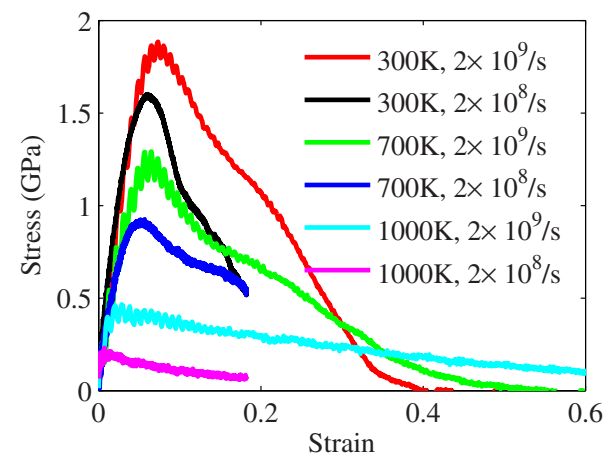

FIG. 3. (Color online) Uniaxial stress vs nominal strain curves for a nanowire with diameter $15 \mathrm{~nm}$ and length $57 \mathrm{~nm}$ deformed at strain rates $2 \times 10^{8}$ and $2 \times 10^{9} \mathrm{~s}^{-1}$ at 300,700 , and $1000 \mathrm{~K}$. The sample was only strained to $20 \%$ at the strain rate of $2 \times 10^{8} \mathrm{~s}^{-1}$. volume causes a dilatation of the amorphous network, which is balanced by a flux of atoms from the surface. The dilatation leads to a steady increase in the length of the wire, while wire radius decreases as atoms at the surface rearrange to accommodate the free-volume flux so that the overall volume of the specimen remains approximately constant.

Direct evidence for nucleation and diffusion of free volume can be seen in Fig. 5, which shows the arrangement of atoms in a cross section of a nanowire with $15 \mathrm{~nm}$ diameter, both (a) before deformation and (b) after 60\% strain at 1000 $\mathrm{K}$ and at the strain rate of $2 \times 10^{9} \mathrm{~s}^{-1}$. A substantial increase in free volume can be seen near the center of the wire. In addition, atoms near the surface of the wire have clearly migrated a significant distance toward the center of the wire. Figure 6, which shows the radial mean-square distance vs. time for atoms in the slice of the specimen shown in Fig. 2, gives a more direct measure of the rate of diffusion in the specimen. Quantitative measures of diffusivity will be given in Sec. IV.

To demonstrate that hydrostatic stress can readily nucleate free volume in amorphous solids and to quantify the rate of free-volume nucleation as a function of hydrostatic stress, we have conducted a series of simulations in which a cubic

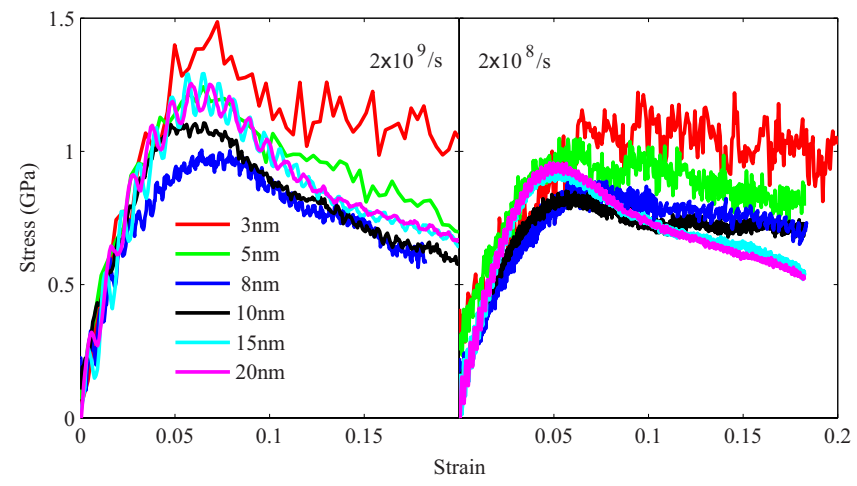

FIG. 4. (Color online) Uniaxial stress vs nominal strain curves for nanowires with diameters from 3 to $20 \mathrm{~nm}$ at a temperature 700 $\mathrm{K}$ and at strain rates $2 \times 10^{9} \mathrm{~s}^{-1}$ (left) and $2 \times 10^{8} \mathrm{~s}^{-1}$ (right). 

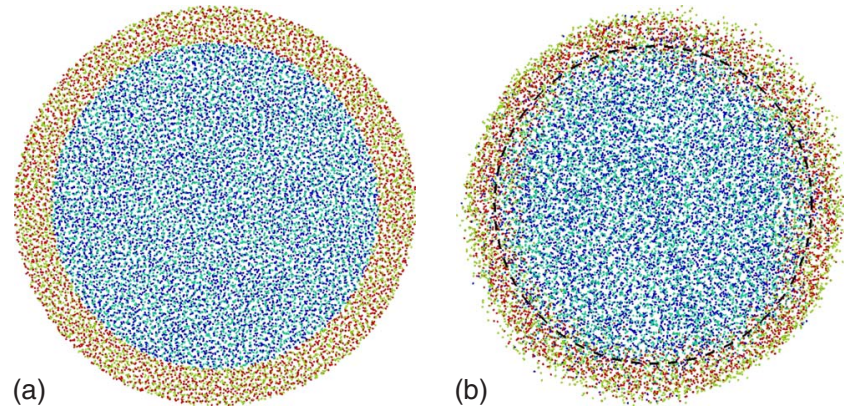

FIG. 5. (Color online) Cross-section showing the arrangement of atoms through the axis of a $15 \mathrm{~nm}$ nanowire. Atoms in the peripheral of the initial specimen are colored for $\mathrm{Cu}$ and red for $\mathrm{Zr}$ and those inside are colored in blue and cyan for $\mathrm{Cu}$ and $\mathrm{Zr}$. respectively. (a) Initial configuration. (b) After deformation at $1000 \mathrm{~K}$ and strain rate $2 \times 10^{9} \mathrm{~s}^{-1}$ to a nominal strain of $60 \%$. Dashed line indicates the original boundary of two groups.

specimen of amorphous $\mathrm{Cu}_{50} \mathrm{Zr}_{50}$ with side length $15 \mathrm{~nm}$ and containing 187731 atoms (cut from the cooled cuboid) was subjected to constant tensile stress normal to its faces. Periodic boundary conditions are applied to the cell. The sample is first relaxed at a given temperature and null pressure for 200 ps and then loaded by hydrostatic tension with a loading rate of $1 \mathrm{MPa} / \mathrm{ps}$. The pressure is held fixed once it reaches $300 \mathrm{MPa}$. The volume of the simulation cell at constant pressure is then computed as a function of time. The resulting increasing volumetric strain in the cube at $300 \mathrm{MPa}$ and 700 $\mathrm{K}$ is plotted in Fig. 7. We observe a transient increase in the volume of the simulation cell as the free-volume concentration increases to a new equilibrium value. This behavior is characterized using phenomenological constitutive equations described in the next section.

\section{ANALYTICAL MODEL OF DEFORMATION BY COUPLED DIFFUSION AND VISCOUS FLOW IN NANOWIRES}

We show next that the qualitative trends observed in our molecular dynamics simulations can be predicted by an ana-

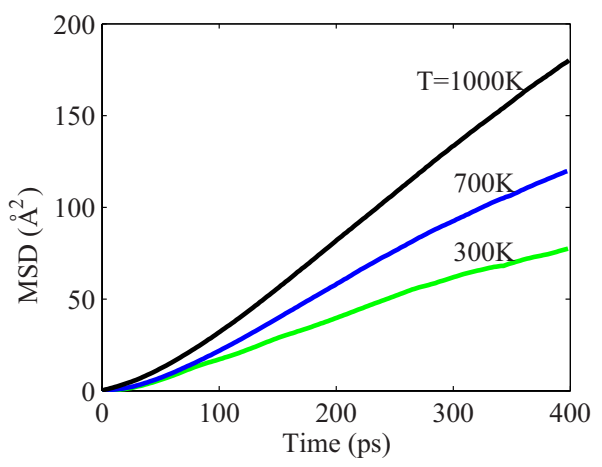

FIG. 6. (Color online) MSD vs time for atoms in the slice (Fig. 2) of the $15 \mathrm{~nm}$ wire at several temperatures during straining at 2 $\times 10^{8} \mathrm{~s}^{-1}$. To obtain the MSD, the effects of deformation were subtracted but no distinction is made between $\mathrm{Cu}$ and $\mathrm{Zr}$ atoms or between atoms inside the wire and those on the wire surface.

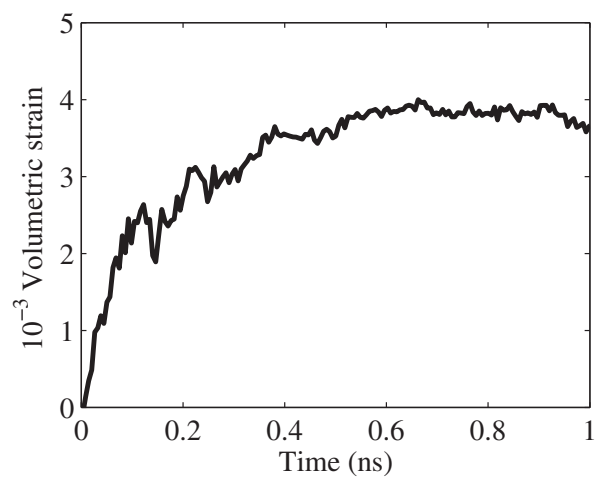

FIG. 7. Variation of volumetric strain with time in a cubic specimen of $\mathrm{Cu}_{50} \mathrm{Zr}_{50}$ subjected to hydrostatic tension of $300 \mathrm{MPa}$ at 700 $\mathrm{K}$. The deformation while the pressure is ramped up to $300 \mathrm{MPa}$ is not shown. The increase in volume occurs at constant pressure.

lytical model that accounts for combined deformation by viscous flow and nucleation of free volume. Our model is based on a highly simplified, and at best incomplete, model of freevolume nucleation and diffusion in amorphous metallic nanowires, but it is nevertheless helpful to demonstrate the phenomenon of interest.

We consider a cylindrical NW with radius $R$, which is subjected to a prescribed macroscopic strain rate $\dot{\varepsilon}_{z z}$ parallel to its axis. Motivated by the MD simulations described in the preceding section, we describe the deformation and constitutive behavior of the wire using the general framework of Huang et al. ${ }^{23}$ We account for three mechanisms that may cause the wire to change its shape: (i) viscous shearing, (ii) nucleation of free volume, and (iii) free-volume diffusion (or, equivalently, diffusion of atoms into the additional space that results from free-volume nucleation). The first two deformation mechanisms are described by a strain rate tensor, which quantifies the relative velocities between atoms in a representative volume element (RVE). We regard the RVE to be a region of material that is slightly greater than the short-range dense-cluster packing ${ }^{24,25}$ so that atoms can pass through channels between such clusters. Diffusion involves exchange of free volume and atoms between adjacent RVEs. This exchange does not change the size or shape of the elements and therefore does not directly give rise to a strain rate in the RVE. The total strain rate therefore consists of a deviatoric plastic part $\dot{\varepsilon}_{i j}^{p}$ (caused by viscous shearing), together with a dilatational part (caused by the generation of free volume)

$$
\dot{\varepsilon}_{i j}=\dot{\varepsilon}_{i j}^{p}+\dot{\xi}_{n} \delta_{i j} / 3 \text {. }
$$

Elastic strains have been neglected for simplicity.

The deviatoric plastic strain rate is related to stress by a linear viscous constitutive equation

$$
\dot{\varepsilon}_{i j}^{p}=\dot{\varepsilon}_{0} \frac{3}{2} \frac{S_{i j}}{\sigma_{0}},
$$

where $S_{i j}=\sigma_{i j}-\sigma_{k k} \delta_{i j} / 3$ is the deviatoric stress and $\dot{\varepsilon}_{0}$ and $\sigma_{0}$ are material properties. We have neglected the influence of free volume on the viscosity of the material and have assumed linear viscous behavior to simplify calculations. 
These are, of course, gross oversimplifications. It would be possible to generalize our calculations, but the governing equations would need to be solved numerically.

Free-volume nucleation in Eq. (1) is characterized using a phenomenological constitutive equation. This approach is frequently used in models of metallic glass, but existing models differ widely in their details. In some cases, freevolume nucleation is assumed to be driven by shear stresses $^{26}$ or to be generated as a consequence of the deviatoric plastic strain, ${ }^{27}$ while other models account for the effects of hydrostatic stress. ${ }^{28,29}$ Motivated by the MD simulation results shown in Fig. 7, we assume that nucleation is a thermally activated process driven by the change in free energy per atom resulting from an increase in free volume

$$
\Delta \mu=k T \log \left(\xi / \xi_{e}\right)-\Omega_{d} \sigma_{k k} / 3,
$$

where $\xi_{e}$ is the equilibrium free-volume concentration in a large, stress-free specimen and a function of temperature, $k$ is the Boltzmann constant, $T$ is temperature, and $\xi=(\Omega$ $\left.-\Omega_{d}\right) / \Omega_{d}$ represents the excess free-volume concentration, with $\Omega$ being the volume per atom and $\Omega_{d}$ the volume per atom in a dense randomly packed solid. The first term represents an entropic contribution to the free-energy change, while the second is the work done by the applied stress. The rate of free-volume nucleation is approximated as

$$
\dot{\xi}_{n}=-\frac{\dot{X}_{0}}{k T} \Delta \mu
$$

where $\dot{X}_{0}$ is a temperature-dependent rate constant. Note that Eq. (3) predicts that the equilibrium free-volume concentration increases exponentially with hydrostatic stress. In addition, integrating Eqs. (3) and (4) predicts that a step increase in hydrostatic stress results in a transient increase in volume to a new equilibrium value, consistent with the MD simulations shown in Fig. 7.

The diffusion of free volume is driven by a chemical potential

$$
\mu=\mu_{0}+k T \log \left(\xi / \xi_{e}\right),
$$

where $\mu_{0}$ is a reference chemical potential. Note that the driving force for diffusion is not directly dependent of stress, since we assume that the externally applied stresses do no work during free-volume diffusion (which does not induce a strain). In the limit of infinitely fast nucleation, the condition $\Delta \mu=0$ yields $\mu=\mu_{0}+\Omega_{d} \sigma_{k k} / 3$, as used for diffusion in polycrystalline metals. In polycrystalline counterparts, we often assume infinitely fast nucleation: the thermal equilibrium $\xi$ $=\xi_{e} \exp \left(-\Omega_{d} \sigma_{k k} / 3 k T\right)$ is always satisfied. Hence, the driving force for diffusion of vacancies is equivalent to pressure gradient. In metallic glass, thermal equilibrium is not guaranteed and we adopted the original formulation of entropydriven diffusion, as shown in Eq. (5). It is still pressure dependent since $\xi$ depends on pressure although there is no simple relation between free-volume concentration and pressure seen in the thermal equilibrium condition. The freevolume flux is proportional to the gradient of chemical potential

$$
\mathbf{j}=-\frac{D}{k T} \nabla \mu,
$$

where $D$ is a temperature-dependent diffusivity. Finally, mass conservation requires that

$$
\frac{\partial \xi}{\partial t}=-\nabla \cdot \mathbf{j}+\dot{\xi}_{n} .
$$

This description of free-volume nucleation and diffusion has the advantage that analytical expressions for the stress and deformation in the NW can easily be calculated. The model used here would not give an accurate description of the behavior of a macroscopic specimen of metallic glass and is therefore at best incomplete.

The deformation induces an axially symmetric stress state $\left(\sigma_{r r}, \sigma_{\theta \theta}, \sigma_{z z}\right)$. The stresses satisfy the equilibrium equation $\partial \sigma_{i j} / \partial x_{i}=0$.

The model is completed by boundary conditions for stress and free-volume concentration at the surface of the wire. We assume that the wire has isotropic surface stress $\gamma$, which can be visualized as an isotropic membrane tension acting in a vanishingly thin layer at the surface of the specimen. The stresses in the solid material immediately below this membrane layer must satisfy

$$
\sigma_{i j} n_{j}=-\gamma\left(\kappa_{1}+\kappa_{2}\right) n_{i}
$$

for mechanical equilibrium, where $n_{i}$ are the components of a unit vector normal to the surface, and $\kappa_{1}$ and $\kappa_{2}$ are the principal curvatures of the surface, with the convention that a convex surface has positive curvature. We regard the surface as a perfect sink for free volume, so that the excess freevolume concentration must remain at its equilibrium value at the surface. This leads to a Gibbs-Thompson condition of the form

$$
\xi=\xi_{e} \exp \left(-\frac{\Omega_{d} \gamma\left(\kappa_{1}+\kappa_{2}\right)}{k T}\right),
$$

where we have assumed that the solid's surface stress and surface energy are equal. Finally, mass conservation at the surface yields the normal velocity of the surface relative to the underlying material as

$$
v_{n}=-j_{i} n_{i} .
$$

It is straightforward to solve Eqs. (1)-(9), together with the equilibrium equation, to determine the steady-state stress, strain rate, and velocity fields in the wire, as well as the free-volume concentration. To express the solution in the simplest form, it is convenient to introduce a characteristic length scale

$$
\rho=\sqrt{\frac{4 \sigma_{0} \Omega_{d} D}{9 \dot{\varepsilon}_{0} k T}+\frac{D}{\dot{X}_{0}}} .
$$

This length scale controls the thickness of the layer at the surface of the wire where diffusion dominates over viscous shearing and free-volume nucleation. It appears in models for both amorphous NWs (Appendix A) and thin plates or membrane (Appendix B). We then introduce dimensionless 
wire radius and position $\widetilde{R}=R / \rho, \tilde{r}=r / \rho$. In terms of these variables, the stresses are (see Appendix A for details)

$$
\begin{aligned}
& p=-\frac{\gamma}{R}+\frac{3 \dot{\varepsilon}_{z z} \widetilde{R}}{2} \frac{1}{(2+\Pi) I_{0}(\widetilde{R}) \widetilde{R}-3 I_{1}(\widetilde{R})}\left\{\frac{k T I_{0}(\widetilde{R})}{\dot{X}_{0} \Omega_{d}}\right. \\
& \left.+\frac{4 \sigma_{0}}{9 \dot{\varepsilon}_{0}}\left[I_{0}(\widetilde{R})-I_{0}(\widetilde{r})\right]\right\} \text {, } \\
& \sigma_{r r}=\frac{\dot{\varepsilon}_{z z} \sigma_{0} \tilde{R}}{\dot{\varepsilon}_{0}} \frac{1}{(2+\Pi) I_{0}(\widetilde{R}) \widetilde{R}-3 I_{1}(\widetilde{R})}\left(\frac{I_{1}(\widetilde{R})}{\widetilde{R}}-\frac{I_{1}(\widetilde{r})}{\widetilde{r}}\right)-\frac{\gamma}{R}, \\
& \sigma_{\theta \theta}=p+\frac{\dot{\varepsilon}_{z z} \sigma_{0} \widetilde{R}}{3 \dot{\varepsilon}_{0}} \frac{1}{(2+\Pi) I_{0}(\widetilde{R}) \widetilde{R}-3 I_{1}(\widetilde{R})}\left(\frac{3 I_{1}(\widetilde{r})}{\widetilde{r}}-I_{0}(\widetilde{r})\right) \\
& -\frac{\dot{\varepsilon}_{z z} \sigma_{0}}{3 \dot{\varepsilon}_{0}}, \\
& \sigma_{z z}=p-\frac{\dot{\varepsilon}_{z z} \sigma_{0} \widetilde{R}}{3 \dot{\varepsilon}_{0}} \frac{I_{0}(\widetilde{r})}{(2+\Pi) I_{0}(\widetilde{R}) \widetilde{R}-3 I_{1}(\widetilde{R})}+\frac{2 \dot{\varepsilon}_{z z} \sigma_{0}}{3 \dot{\varepsilon}_{0}},
\end{aligned}
$$

where $I_{n}$ is the modified Bessel function of the first kind of order $n$ and

$$
\Pi=\frac{9 \dot{\varepsilon}_{0} k T}{2 \sigma_{0} \dot{X}_{0} \Omega_{d}}
$$

is a dimensionless parameter, which quantifies the rate of viscous shearing compared to the free-volume nucleation rate.

The flux of free volume is radial, with distribution

$$
j_{r}=\frac{3 \dot{\varepsilon}_{z z} \rho \tilde{R}}{2} \frac{I_{1}(\widetilde{r})}{(2+\Pi) I_{0}(\widetilde{R}) \widetilde{R}-3 I_{1}(\widetilde{R})} .
$$

The strain rate distribution is

$$
\begin{gathered}
\dot{\varepsilon}_{r r}=\frac{3 \dot{\varepsilon}_{z z} \widetilde{R}}{2} \frac{1}{(2+\Pi) I_{0}(\widetilde{R}) \tilde{R}-3 I_{1}(\widetilde{R})}\left(I_{0}(\widetilde{r})-\frac{I_{1}(\widetilde{r})}{\widetilde{r}}\right)-\frac{\dot{\varepsilon}_{z z}}{2}, \\
\dot{\varepsilon}_{\theta \theta}=\frac{3 \dot{\varepsilon}_{z z} \widetilde{R}}{2} \frac{1}{(2+\Pi) I_{0}(\widetilde{R}) \tilde{R}-3 I_{1}(\widetilde{R})} \frac{I_{1}(\widetilde{r})}{\widetilde{r}}-\frac{\dot{\varepsilon}_{z z}}{2}, \\
\dot{\varepsilon}_{k k}=\frac{3 \dot{\varepsilon}_{z z} \widetilde{R}}{2} \frac{I_{0}(\widetilde{r})}{(2+\Pi) I_{0}(\widetilde{R}) \tilde{R}-3 I_{1}(\widetilde{R})} .
\end{gathered}
$$

The velocity field associated with this strain rate distribution has the form
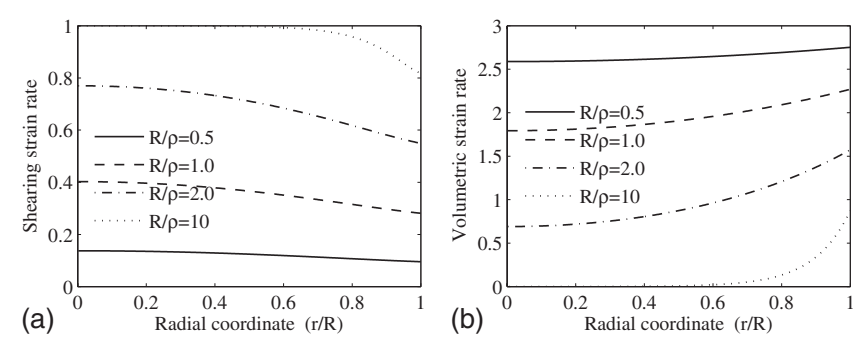

FIG. 8. Variation of steady-state strain rate along the radial direction of a nanowire with varying ratios of wire radius $R$ to characteristic material length $\rho$. (a) Shear (deviatoric) strain rate $\left(\sqrt{2 \dot{\varepsilon}_{i j}^{p} \dot{\varepsilon}_{i j}^{p} / 3}\right) / \dot{\varepsilon}_{z z}$ and (b) volumetric strain rate $\dot{\varepsilon}_{k k} / \dot{\varepsilon}_{z z}$. Results are shown for $\gamma=\Pi=0$.

$\dot{u}_{r}=\frac{3 \dot{\varepsilon}_{z z} \rho \widetilde{R}}{2} \frac{I_{1}(\widetilde{r})}{(2+\Pi) I_{0}(\widetilde{R}) \widetilde{R}-3 I_{1}(\widetilde{R})}-\frac{\dot{\varepsilon}_{z z} \rho \widetilde{r}}{2} \quad$ and $\quad \dot{u}_{z}=\dot{\varepsilon}_{z z} z$

Note that material particles have a positive instantaneous radial velocity due to the dilatational strain associated with free-volume nucleation. At the surface of the wire, the flux of free volume causes material at the surface to be annihilated. The velocity of the surface with respect to a fixed laboratory frame follows as $V_{n}=\dot{u}_{r}(R)-j_{r}(R)=-\dot{\varepsilon}_{z z} R / 2$, as expected from volume conservation.

Finally, the net axial force on the wire follows as

$$
F=2 \pi R \gamma+\int_{0}^{R} \sigma_{z z} 2 \pi r d r
$$

where the first term represents a contribution to the force from the membrane tension resulting from surface stress. The integral can be easily evaluated to yield

$$
\frac{F}{\pi R^{2}}=\frac{\gamma}{R}+\frac{\dot{\varepsilon}_{z z} \sigma_{0}}{\dot{\varepsilon}_{0}}\left(1-\frac{I_{1}(\widetilde{R})}{(2+\Pi) I_{0}(\widetilde{R}) \widetilde{R}-3 I_{1}(\widetilde{R})}\right) .
$$

As expected, the force is proportional to the strain rate. In the absence of an external force, surface stress causes the wire to contract. Note that $I_{1}(\widetilde{R}) \rightarrow 0$ for large $\widetilde{R}$, so for thick wires, we recover the expected viscous creep behavior. The nominal flow stress decreases as wire radius is reduced.

Important features of the solution are illustrated in Figs. 8 and 9. Figure 8 shows the distributions of deviatoric and volumetric strain rates in wires with various normalized radii $R / \rho$, while Fig. 9 shows the corresponding distributions of axial stress in the wire. In wires with a large $R / \rho$, the volumetric strain rate is zero except in a thin layer near the surface, while the deviatoric strain rate is essentially uniform. Under these conditions, the wire deforms primarily by viscous shearing and the axial stress is essentially constant, except for a boundary layer near the wire surface. As the wire radius is progressively reduced, the volumetric strain rate increases, while the deviatoric strain rate decreases. This indicates that diffusion provides an increasing contribution to the strain rate in the wire. As a result, the stress (at fixed applied strain rate) progressively decreases. The transition 


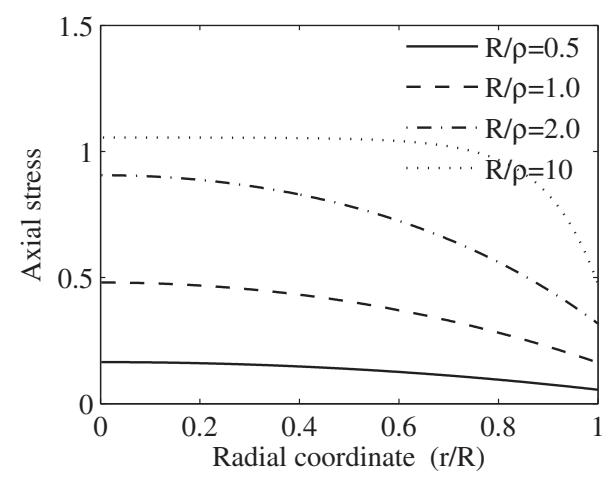

FIG. 9. Variation of steady-state normalized axial stress $\left(\sigma_{z z} \dot{\varepsilon}_{0} / \sigma_{0} \dot{\varepsilon}_{z z}\right)$ along the radial direction of a nanowire with varying ratios of wire radius $R$ to characteristic material length. Results are shown for $\gamma=\Pi=0$.

from primarily viscous shearing to diffusional deformation occurs for $R / \rho \approx 1$. In the limit $R / \rho \rightarrow 0$, viscous creep can be neglected and the expressions for stress, strain rate, and flux of free volume reduce to simple forms (see Appendix A)

$$
\begin{gathered}
\dot{\varepsilon}_{r r}=\dot{\varepsilon}_{\theta \theta}=\dot{\varepsilon}_{z z}, \\
\sigma_{z z}=-\frac{\gamma}{R}+\frac{3 \dot{\varepsilon}_{z z} k T}{\dot{X}_{0} \Omega_{d}}+\frac{9 k T \dot{\varepsilon}_{z z} R^{2}}{4 \Omega_{d} D}\left(1-\frac{r^{2}}{R^{2}}\right), \quad \sigma_{r r}=\sigma_{\theta \theta}=-\frac{\gamma}{R}, \\
j_{r}=3 \dot{\varepsilon}_{z z} r / 2 \quad j_{\theta}=j_{z}=0 .
\end{gathered}
$$

It is worth noting also that for the limiting values of $R / \rho \rightarrow 0, \gamma=0$, and $\dot{X}_{0} \rightarrow \infty$, the axial force-v-strain rate relation reduces to

$$
\dot{\varepsilon}_{z z}=\frac{8 D \Omega_{d}}{9 k T R^{2}} \frac{F}{\pi R^{2}} .
$$

This result resembles the strain rate-v-stress relation for a solid that deforms by Nabarro-Herring creep: the creep rate by bulk diffusion in an amorphous wire of radius $R$ is equivalent to that in a polycrystal with an average grain size of $3.35 R$.

To compare the predictions of the analytical model with MD simulations, it is convenient to introduce a normalized strain rate defined as

$$
\dot{E}=\frac{\dot{\varepsilon}_{z z} \sigma_{0}}{\dot{\varepsilon}_{0}\left(F / \pi R^{2}\right)}=\frac{1}{\frac{B}{\widetilde{R}}+\left(1-\frac{I_{1}(\widetilde{R})}{(2+\Pi) I_{0}(\widetilde{R}) \tilde{R}-3 I_{1}(\widetilde{R})}\right)},
$$

where $B=\gamma \dot{\varepsilon}_{0} /\left(\rho \sigma_{0} \dot{\varepsilon}_{z z}\right)$ is a dimensionless surface energy. Figure 10 shows the variation of normalized strain rate as a function of wire radius for various values of the dimensionless material parameters $\Pi$ and $B$.

To interpret these predictions, consider first the behavior in the limit of vanishing surface tension $[B=0$, Fig. 10(a)]. In this case, we observe three general regimes of behavior. In wires with large radius, deformation is dominated by viscous
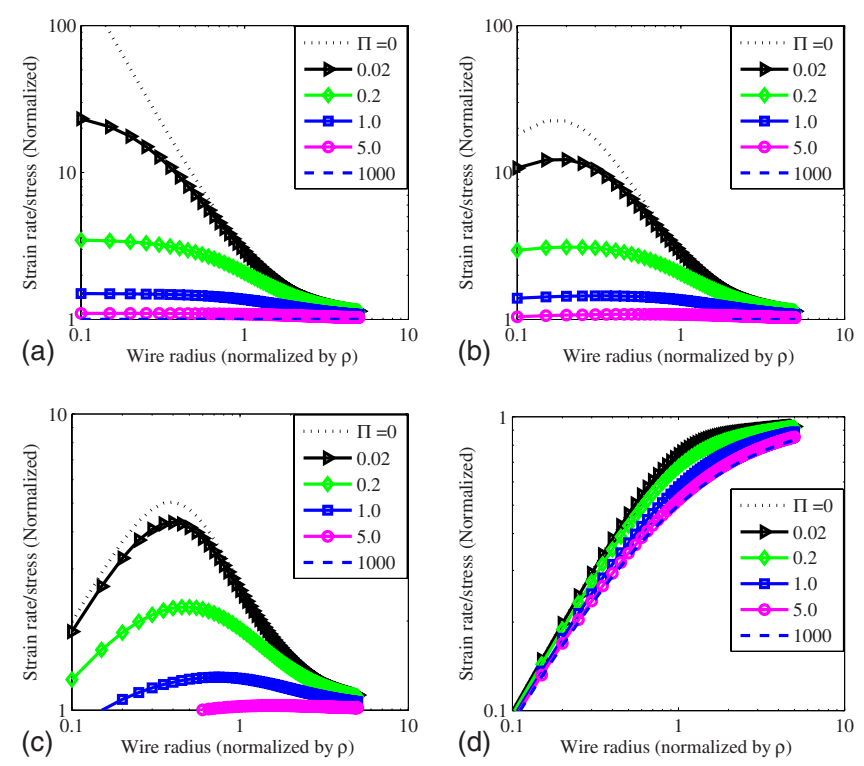

FIG. 10. (Color online) Dependence of $\dot{\varepsilon}_{z z} /\left(F / \pi R^{2}\right)$ (normalized by its value for sufficiently large wire) vs wire radius for different B: (a) $B=0$, (b) $B=0.1$, (c) $B=1$, and (d) $B=20$. In (d), note the linear scaling of +1 for small $R / \rho$.

flow and the normalized strain rate approaches $\dot{E}=1$. As the wire radius decreases, the normalized strain rate initially increases (or equivalently, the flow stress decreases). The rate of increase is determined by the rate of viscous shearing compared to free-volume nucleation (parameterized by П). If free volume is nucleated easily (small $\Pi$ ), we observe that the normalized strain rate increases as $\widetilde{R}^{-2}$ [see Fig. 10(a)], consistent with Eq. (17). For larger values of $\Pi$, the strain rate initially increases and then approaches a constant asymptote [see Fig. 10(a)]. In the limit $R \rightarrow 0$, the strain rate is controlled by the rate of free-volume nucleation.

For larger values of $B$ [Fig. 10(d)], another regime of behavior emerges in the limit of small wire radius. In this regime, the normalized strain rate is dominated by the effects of surface stress, which leads to a decreasing normalized strain rate (or equivalently, an increasing flow stress) with decreasing wire radius. In the limit of vanishing wire radius, the normalized strain rate is proportional to $\widetilde{R}$ in this regime. Depending on the value of $B$ [see Figs. 10(a) $-10(\mathrm{~d})$ ], behavior can be dominated either by surface stress effects [Fig. 10(d)] or by diffusion [Fig. 10(a)].

Our analytical calculations indicate that the minimum flow stress (or maximum normalized strain rate) observed in MD simulations can be explained qualitatively by the competing effects of surface stress and free-volume nucleation and diffusion in the NW. In the next section, we shall attempt a more quantitative comparison of the analytical model with MD simulation results.

\section{COMPARISON OF ANALYTICAL MODEL AND MD SIMULATIONS}

In this section, we use molecular dynamics simulations to estimate material parameters for the analytical model de- 


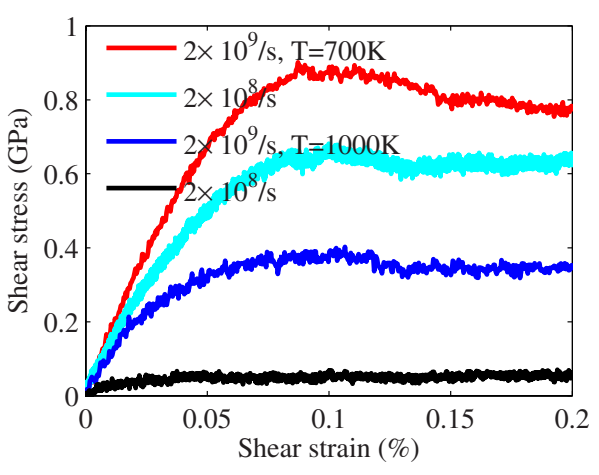

FIG. 11. (Color online) Shear stress vs shear strain at different strain rates and temperatures.

scribed in the preceding section. It would, of course, be possible to fit material parameters directly to the results of our MD simulations of deformation in nanowires. This is not a critical test of the analytical model, however. Instead, we calibrate the parameters by means of a series of MD simulations in which periodic unit cells of the metallic glass are subjected to prescribed loading. Naturally, in view of the approximations made to simplify analytical calculations, a perfect fit is impossible to achieve, but parameter values that best approximate material behavior can be estimated.

The analytical calculations contain four material parameters: the viscosity $\sigma_{0} / \dot{\varepsilon}_{0}$, the diffusion coefficient $D$, the free-volume nucleation rate $\dot{X}_{0}$, and the surface energy $\gamma$. The viscosity can be estimated by applying simple shearing deformation to a periodic unit cell, while the diffusion coefficient can be estimated from the mean-square displacement of atoms. The free-volume nucleation rate can be estimated by subjecting a periodic unit cell to prescribed hydrostatic stress. The surface energy is difficult to compute directly and we will simply assume a value of $\gamma=0.2 \mathrm{~J} / \mathrm{m}^{2}$ when comparing MD simulations to the analytical model. The procedure to compute relevant material parameters is described in more detail in the following sections.

\section{A. Viscosity $\sigma_{0} / \dot{\varepsilon}_{0}$}

Viscosities at different strain rates and temperatures for $\mathrm{Cu}_{50} \mathrm{Zr}_{50}$ were estimated by subjecting a periodic cube of side length $15 \mathrm{~nm}$ to prescribed shearing displacements. Representative shear stress-v-shear strain curves are shown in Fig. 11. The viscosity at each temperature and strain rate is estimated as $\sigma_{0} / \dot{\varepsilon}_{0}=\tau / \dot{\varepsilon}_{x z}$, where $\tau$ is the steady-state shear

TABLE I. Shear stress vs. shear strain at different strain rates and temperatures.

\begin{tabular}{cccc}
\hline $\begin{array}{c}\text { Temperature } \\
(\mathrm{K})\end{array}$ & $\begin{array}{c}\text { Rate } \dot{\varepsilon}_{x z} \\
(1 / \mathrm{s})\end{array}$ & $\begin{array}{c}\text { Shear strength } \tau \\
(\mathrm{MPa})\end{array}$ & $\begin{array}{c}\text { Viscosity } \\
(\mathrm{Pa} \mathrm{s})\end{array}$ \\
\hline \multirow{2}{*}{700} & $2 \times 10^{8}$ & 620 & 3.1 \\
& $2 \times 10^{9}$ & 870 & 0.44 \\
\multirow{2}{*}{1000} & $2 \times 10^{8}$ & 50 & 0.25 \\
& $2 \times 10^{9}$ & 380 & 0.19 \\
\hline \hline
\end{tabular}

TABLE II. Estimated diffusivity values for the model amorphous metal at several temperatures.

\begin{tabular}{lcc}
\hline \hline Temperature $(\mathrm{K})$ & 700 & 1000 \\
Diffusivity $\left(\mathrm{m}^{2} / \mathrm{s}\right)$ & $30 \times 10^{-10}$ & $50 \times 10^{-10}$ \\
\hline \hline
\end{tabular}

strength and $\dot{\varepsilon}_{x z}$ the applied shearing rate: the results are listed in Table I. The linear viscous approximation improves with increasing temperature and is an excellent approximation at $1000 \mathrm{~K}$.

\section{B. Diffusivity}

The diffusivity at a given temperature in an amorphous nanowire was estimated by tracking the mean-square displacement (MSD) of atoms in a small slice in the middle of the wire during their deformation at $2 \times 10^{8} / \mathrm{s}$. Diffusivities at several temperatures listed in Table II are obtained from the $15 \mathrm{~nm}$ wire. The effects of deformation are subtracted but no distinction is made for atoms inside the wire and those on the wire surface in calculating the MSD.

\section{Free-volume nucleation parameter $\dot{X}_{0}$}

We have estimated the parameter $\dot{X}_{0}$ (which characterizes the rate of free-volume nucleation) by subjecting a periodic unit cell of the amorphous solid to a constant prescribed pressure and computing the volumetric strain rate of the cell. Typical results of these calculations are shown in Fig. 7. The nucleation rate $\dot{\xi}_{n}$ at time $t=0$ is estimated from the slope of the volume-v-time curves during the first $400 \mathrm{~ns}$ after the pressure reaches its maximum value and $\dot{X}_{0}$ is then estimated as $\dot{X}_{0} \approx-\dot{\xi}_{n} k T / p \Omega_{d}$, where $p$ is the applied pressure and $\Omega_{d}$ $\approx 1.81 \times 10^{-29} \mathrm{~m}^{3}$ is the atomic volume of $\mathrm{Cu}_{50} \mathrm{Zr}_{50}$. Tables III and IV listed the free-volume nucleation rate $\dot{\xi}_{n}$ and the corresponding value of $\dot{X}_{0}$ at several temperatures and pressures.

\section{Comparison of MD simulations and analytical model}

Given values for the material parameters $\sigma_{0} / \dot{\varepsilon}_{0}$ (viscosity), $\dot{X}_{0}, D$, and $\gamma$, we can proceed to calculate parameters $\{\rho, B, \Pi\}$ in Eq. (18). We use the nucleation rates at $p$ $=300 \mathrm{MPa}$ and the viscosities at the shearing strain rate of $2 \times 10^{8} / \mathrm{s}$ and estimate the average atomic volume $\Omega_{d}$ for $\mathrm{Cu}_{50} \mathrm{Zr}_{50}$ as $1.81 \times 10^{-29} \mathrm{~m}^{3}$ from our MD simulations. The

TABLE III. Free-volume nucleation rate at several temperatures and pressures. The nucleation rates are obtained by linearizing corresponding volumetric strain vs. time curves (similar to that in Fig. 7) from 0 to $400 \mathrm{ps}$.

\begin{tabular}{lcccc}
\hline \hline$\dot{\xi}_{n}$ & $\begin{array}{c}p=100 \\
(\mathrm{MPa})\end{array}$ & $\begin{array}{c}p=300 \\
(\mathrm{MPa})\end{array}$ & $\begin{array}{c}p=400 \\
(\mathrm{MPa})\end{array}$ & $\begin{array}{c}p=500 \\
(\mathrm{MPa})\end{array}$ \\
\hline$T=700 \mathrm{~K}$ & $2.5 \times 10^{6}$ & $6.8 \times 10^{6}$ & $8.2 \times 10^{6}$ & $1.4 \times 10^{7}$ \\
$T=1000 \mathrm{~K}$ & $3.9 \times 10^{6}$ & $7.2 \times 10^{6}$ & $9.5 \times 10^{6}$ & $1.7 \times 10^{7}$ \\
\hline \hline
\end{tabular}


TABLE IV. Free-volume nucleation parameter $\dot{X}_{0}$ at several temperatures and pressures.

\begin{tabular}{lcccc}
\hline \hline$\dot{X}_{0}$ & $\begin{array}{c}P=100 \\
(\mathrm{MPa})\end{array}$ & $\begin{array}{c}P=300 \\
(\mathrm{MPa})\end{array}$ & $\begin{array}{c}P=400 \\
(\mathrm{MPa})\end{array}$ & $\begin{array}{c}P=500 \\
(\mathrm{MPa})\end{array}$ \\
\hline$T=700 \mathrm{~K}$ & $1.2 \times 10^{7}$ & $1.1 \times 10^{7}$ & $9.9 \times 10^{6}$ & $1.4 \times 10^{7}$ \\
$T=1000 \mathrm{~K}$ & $2.7 \times 10^{7}$ & $1.7 \times 10^{7}$ & $1.6 \times 10^{7}$ & $2.3 \times 10^{7}$ \\
\hline \hline
\end{tabular}

resulting values of the characteristic radius $\rho$ at several temperatures are listed in Table V. The characteristic radius decreases with increasing temperature because the viscosity of the solid varies more rapidly with temperature than the diffusivity: this trend appears to be consistent with experimental data for metallic glasses, as discussed further in the next section. The dimensionless parameter $\Pi$ is on the order of 10 and $B$ is on the order of 0.1 .

The quantity $\dot{\varepsilon}_{z z} /\left(F / \pi R^{2}\right)$ computed from the MD simulations is shown as a function of wire radius in Fig. 12. The analytical results are also shown for $B=0.1, \rho=15 \mathrm{~nm}$, and $\Pi=0.3$. The analytical model clearly predicts a trend that agrees with the MD simulation data. However, the value of $\Pi$ required to fit the MD simulations is more than 1 order of magnitude smaller than the value estimated from the periodic unit-cell calculations described in the preceding section. The analytical model greatly oversimplifies the description of free-volume nucleation in the metallic glass. The most likely explanation for the discrepancy is that free-volume nucleation is accelerated under combined shearing and hydrostatic stress. Since the model was calibrated under purely hydrostatic loading, $\Pi$ is underestimated.

\section{APPLICATION TO $\mathrm{Zr}_{41.2} \mathrm{Ti}_{13.8} \mathrm{Cu}_{12.5} \mathrm{Ni}_{10.0} \mathrm{Be}_{22.5}$}

Due to the limitations of molecular dynamics, direct numerical simulations are possible only for wires with very small diameters subjected to high strain rates. These conditions would be difficult to achieve in experiments. To examine the implications of our results for more realistic conditions, we have estimated approximate values for the characteristic material length scale $\rho$ at different temperatures for $\mathrm{Zr}_{41.2} \mathrm{Ti}_{13.8} \mathrm{Cu}_{12.5} \mathrm{Ni}_{10.0} \mathrm{Be}_{22.5}$ (Vitreloy 1) for the limiting case $\dot{X}_{0} \rightarrow \infty$. To this end, we have assumed that the viscosity $\eta=\sigma_{0} / \dot{\varepsilon}_{0}$ of Vitreloy 1 can be determined by the Vogel-Fulcher-Tammann (VFT) relation

$$
\eta(T)=\eta_{0} \exp \left(\frac{D^{*} T_{0}}{T-T_{0}}\right),
$$

where $\eta_{0}=4 \times 10^{-5}$ Pa s (Ref. 30) and $D^{*}$ and $T_{0}$ are the fragility parameter and the VFT temperature, respectively. ${ }^{31}$

TABLE V. Critical radius at several temperatures based on the viscosity, diffusivity, and free-volume nucleation parameter $\dot{X}_{0}$ obtained in our MD simulations.

\begin{tabular}{lcc}
\hline \hline Temperature (K) & 700 & 1000 \\
$\rho(\mathrm{nm})$ & $\sim 18$ & $\sim 17$ \\
\hline \hline
\end{tabular}
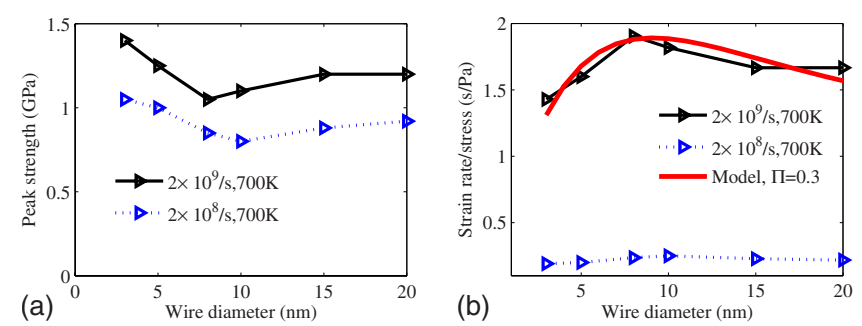

FIG. 12. (Color online) (a) Peak strength vs wire diameter at two strain rates and $700 \mathrm{~K}$. (b) Strain rates-stress vs wire diameter from MD simulations and a fitted curve from our analytical model. To fit the simulation results, we have used $B=0.1, \rho=15 \mathrm{~nm}$, and $\Pi=0.3$.

We take $D^{*}=18.5$ and $T_{0}=412.5 \mathrm{~K}$ for Vitreloy $1 .{ }^{32}$ The VFT relation between temperature and viscosity is in good agreement with experimental data. ${ }^{33}$ The diffusivity is estimated using

$$
D=D_{0} \exp \left(-\frac{Q}{k T}\right)
$$

with $D_{0}=1.82 \times 10^{-11} \mathrm{~m}^{2} / \mathrm{s}$ and $Q=1.05 \mathrm{eV} .^{8}$ These values correspond to the measured diffusivity of $\mathrm{Be}$ atoms in the glass state of Vitreloy $1 .{ }^{8}$ Diffusion of free volume in metallic glass may involve both individual and collective hoppings of atoms and is likely to be faster. The atomic volume $\Omega_{d}$ was estimated as $\Omega_{d}=1.67 \times 10^{-29} \mathrm{~m}^{3}{ }^{34}$ The resulting variation of $\rho$ with temperature is plotted in Fig. 13. We have also estimated the approximate limits of validity of this expression: at low temperatures, viscous creep ceases to be Newtonian and coupling effects between creep and free-volume nucleation will lead to the formation of shear bands. The predicted values are on the order of $100 \mathrm{~nm}$ at temperatures around $575 \mathrm{~K}$. The radius decreases as temperature is reduced because the viscosity of metallic glasses appears to be more sensitive to temperature than the diffusivity. We predict values of $\rho$ up to $1000 \mathrm{~nm}$ at $550 \mathrm{~K}$ but at these temperatures, the Newtonian viscous approximation ceases to be accurate. These estimates suggest that diffusion may play an important role in NWs with radii of order 20-100 nm. Naturally, in view of the approximate nature of our model, the

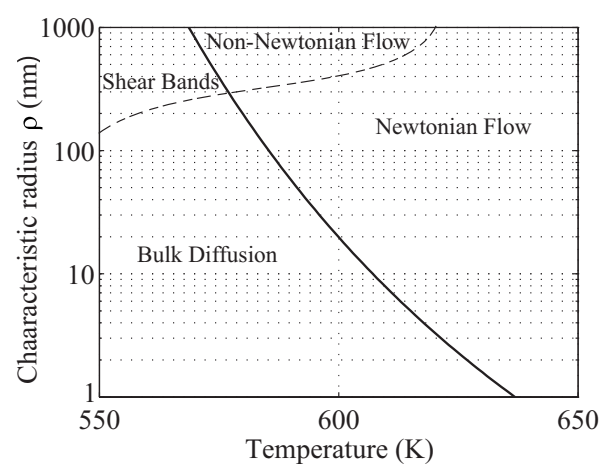

FIG. 13. Dependence of $\rho$ at different temperature for $\mathrm{Zr}_{41.2} \mathrm{Ti}_{13.8} \mathrm{Cu}_{12.5} \mathrm{Ni}_{10.0} \mathrm{Be}_{22.5}$ (solid line) and a speculative deformation map. 
details of the temperature dependence of $\rho$ are unlikely to be accurate.

\section{CONCLUSIONS}

We have used molecular dynamics simulations to compute the flow stress in amorphous $\mathrm{Cu}_{50} \mathrm{Zr}_{50}$ nanowires during axial straining at temperatures near the glass transition. The simulations show a strong sensitivity of flow stress to the diameter of the wire and predict a critical radius that minimizes the flow stress.

A detailed examination of the cross section of the wires shows evidence of significant free-volume nucleation and diffusion within the wire. We suggest that the size effect observed in our MD simulations is a consequence of a transition in deformation mechanism from viscous flow (in large wires) to a combination of viscous flow and diffusion (in small wires). In the diffusional mechanism, free volume is continuously nucleated in the interior of the wire and subsequently diffuses to the free surface.

An approximate analytical model of this process was found to predict a variation of flow stress with wire radius in qualitative agreement with the MD simulations. Quantitative agreement between the analytical model and the MD simulations could only be achieved when the rate of free-volume nucleation in the model was about 10 times greater than that predicted by direct MD simulations, however. A possible explanation for the discrepancy is that the model oversimplifies the mechanism of free-volume nucleation by assuming that free volume is nucleated only by hydrostatic stress and is independent of shearing.

Due to the limitations of MD simulations, direct numerical simulations are possible only for wires with small diameters subjected to large strain rates. Consequently, the analytical calculations were used to estimate the conditions where diffusion is likely to dominate over viscous flow in larger nanowires subjected to lower strain rates. The results suggest that nucleation and diffusion of free volume may provide a mechanism of deformation in metallic glass NWs with radii of order $20-100 \mathrm{~nm}$ at high temperatures ( $>0.6 T_{g}$ ). The model predicts several features that should be verifiable experimentally:

(1) We anticipate an approximately linear relationship between flow strength and strain rate in the diffusional regime.

(2) Diffusion becomes increasingly dominant as wire radius is reduced. In the diffusional regime, the flow strength of the wire at fixed strain rate varies in proportion to the inverse square of the wire radius.

(3) A critical wire radius exists that minimizes flow stress.

(4) For sufficiently large wire radii, the deformation mechanism will transition to predominantly viscous creep. In this regime, the flow stress is independent of wire radius.

(5) The wire radius where deformation transitions between viscous creep and diffusion should decrease with temperature.

Several further developments of the preliminary calculations described here would also be of interest. In particular, it would be instructive to repeat the calculations with more sophisticated constitutive laws governing the nucleation of

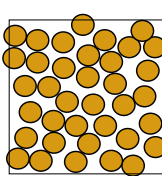

(a)

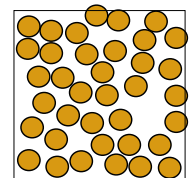

(b)

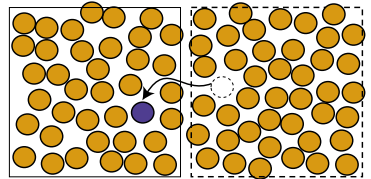

(c)
FIG. 14. (Color online) Schematic illustrating the process of free-volume nucleation and diffusion in an amorphous solid: (a) an initial RVE; (b) expansion of the volume element due to freevolume nucleation; (c) densification due to free-volume diffusion out of the volume element (or, equivalently, diffusion of an atom into the volume element). A neighboring RVE (bound by dashed lines) is also included to show the origin of the blue atom diffusing in the RVE in (b).

free volume, e.g., taking into account the free-volume generation by shearing, ${ }^{24}$ also, non-Newtonian flow should be considered for wider temperature regions, lastly, the effects of free-volume concentration on the shearing response of the glass is of interest and deserves further investigation.

\section{ACKNOWLEDGMENT}

We thank William Nix and Scott Mao for valuable discussions. The simulations reported were performed on NSF TeraGrid resources provided by NCSA under Grant No. DMR090083 and at Alabama Supercomputer Center. The research reported is partly supported by the NSF MRSEC at program (Grant No. DMR-0520651) at Brown University. Y.W. also acknowledges the support from the University of Alabama.

\section{APPENDIX A}

In this appendix, we give a more detailed derivation of the analytical model of deformation in nanowires by coupled diffusion and viscous flow. We begin by clarifying our description of free-volume nucleation and diffusion. Figure 14(a) illustrates a RVE in an amorphous solid. We take a volume element to consist of a set of randomly ordered atoms. The size of a representative element is larger than the short-range dense-cluster packing: the channels between the dense clusters act as sites for nucleation and diffusion of free volume. Free-volume nucleation causes the RVE to increase its size, with a fixed number of atoms in the RVE, as shown in Fig. 14(b). Since the boundaries of the volume element are displaced during this process, it is described as a strain of the underlying material. In contrast, diffusion involves an exchange of free volume (or atoms) between neighboring RVEs, as illustrated in Fig. 14(c): the blue atom added to the RVE comes from the neighboring RVE (bound by dashed lines). This process changes the number of atoms in a volume element without changing its size or shape. Consequently, this process does not induce a strain in the RVE.

RVEs may also experience a change in shape, at constant volume, due to viscous shearing. The shape changes due to free-volume nucleation and viscous shearing are quantified by a strain rate tensor as defined in Eq. (1). We also define a velocity field associated with this strain field, which is re- 
lated to the strain rate components (in cylindrical-polar coordinates) by

$$
\dot{\varepsilon}_{r r}=\frac{\partial \dot{u}_{r}}{\partial r}, \quad \dot{\varepsilon}_{\theta \theta}=\frac{\dot{u}_{r}}{r}, \quad \dot{\varepsilon}_{z z}=\frac{\partial \dot{u}_{z}}{\partial z},
$$

where $\dot{u}_{r}$ is the time derivative of radial displacement measured relative to the current configuration of the solid. Here, the velocity field should be regarded as quantifying the change in shape of the current configuration of the solid, which is taken as the reference configuration for defining the strain rate. The reference configuration evolves due to diffusion.

We now proceed to outline briefly the details of the derivations of Eqs. (11) and (16) in the text.

Derivation of Eq. (11). With Eqs. (1) and (2) and defining $p=\sigma_{k k} / 3$, we rewrite Eq. (1) as

$$
\begin{aligned}
& \dot{\varepsilon}_{r r}=\frac{3 \dot{\varepsilon}_{0}}{2 \sigma_{0}}\left(\sigma_{r r}-p\right)+\frac{\dot{\xi}_{n}}{3}, \\
& \dot{\varepsilon}_{\theta \theta}=\frac{3 \dot{\varepsilon}_{0}}{2 \sigma_{0}}\left(\sigma_{\theta \theta}-p\right)+\frac{\dot{\xi}_{n}}{3}, \\
& \dot{\varepsilon}_{z z}=\frac{3 \dot{\varepsilon}_{0}}{2 \sigma_{0}}\left(\sigma_{z z}-p\right)+\frac{\dot{\xi}_{n}}{3} .
\end{aligned}
$$

It is convenient to express $\dot{\xi}_{n}$ in terms of $\dot{u}_{r}$ and $\dot{\varepsilon}_{z z}$ by summing equations in Eqs. (A2)

$$
\dot{\xi}_{n}=\dot{\varepsilon}_{r r}+\dot{\varepsilon}_{\theta \theta}+\dot{\varepsilon}_{z z} .
$$

With Eq. (A1), we obtain

$$
\dot{\xi}_{n}=\frac{\partial \dot{u}_{r}}{\partial r}+\frac{\dot{u}_{r}}{r}+\dot{\varepsilon}_{z z}
$$

Free-volume nucleation rate $\dot{\xi}_{n}$ is controlled by Eq. (4). Substituting Eqs. (3) and (5) in Eq. (4), we arrive at

$$
\dot{\xi}_{n}=-\dot{X}_{0} \Delta \mu_{v} / k T=\dot{X}_{0}\left(\Omega_{d} p-\mu_{v}\right) / k T .
$$

Now the chemical potential of free volume $\Omega_{d}$ is given as

$$
\mu_{v}=\Omega_{d} p-\frac{k T}{\dot{X}_{0}} \dot{\xi}_{n} .
$$

Using Eqs. (6) and (7) and noting that at steady state, $\partial \xi / \partial t=0$, we see that

$$
\begin{aligned}
& \frac{D \Omega_{d}}{k T} \frac{1}{r} \frac{\partial}{\partial r}\left(r \frac{\partial p}{\partial r}\right)-\frac{D}{\dot{X}_{0}} \frac{1}{r} \frac{\partial}{\partial r}\left[r \frac{\partial}{\partial r}\left(\frac{\partial \dot{u}_{r}}{\partial r}+\frac{\dot{u}_{r}}{r}+\dot{\varepsilon}_{z z}\right)\right] \\
&+\left(\frac{\partial \dot{u}_{r}}{\partial r}+\frac{\dot{u}_{r}}{r}+\dot{\varepsilon}_{z z}\right)=0 .
\end{aligned}
$$

With Eqs. (A1) and (A3), we can also rewrite Eq. (A2a) as

$$
\frac{\partial \dot{u}_{r}}{\partial r}=\frac{3 \dot{\varepsilon}_{0}}{2 \sigma_{0}}\left(\sigma_{r r}-p\right)+\frac{1}{3}\left(\frac{\partial \dot{u}_{r}}{\partial r}+\frac{\dot{u}_{r}}{r}+\dot{\varepsilon}_{z z}\right) .
$$

Recall that the radial component of the equation of equilibrium can be expressed in cylindrical-polar coordinates as

$$
\frac{\partial \sigma_{r r}}{\partial r}+\frac{\sigma_{r r}-\sigma_{\theta \theta}}{r}=0 .
$$

Subtracting Eq. (A2b) from Eq. (A2a) and then using Eq. (A1), we have

$$
r \frac{\partial}{\partial r}\left(\frac{\dot{u}_{r}}{r}\right)=\frac{3 \dot{\varepsilon}_{0}}{2 \sigma_{0}}\left(-r \frac{\partial \sigma_{r r}}{\partial r}\right) .
$$

There are three unknowns $\left\{\dot{u}_{r}, \sigma_{r r}, p\right\}$ in Eqs. (A6), (A7), and (A9), so we can solve these equations together with boundary conditions listed in Eqs. (9) and (10). First, we solve for $\dot{u}_{r}$,

$$
\dot{u}_{r}=c_{1} I_{1}(r / \rho)-\frac{\dot{\varepsilon}_{z z}}{2} r, \quad \text { with } \quad \rho^{2}=\frac{4 D \Omega_{d} \sigma_{0}}{9 k T \dot{\varepsilon}_{0}}+\frac{D}{\dot{X}_{0}},
$$

where $I_{n}$ is a modified Bessel function of the first kind of order $n$ and $c_{1}$ is a constant to be determined. We can now derive $\left\{p, \sigma_{r r}, \dot{\xi}_{n}\right\}$ as

$$
p=-\frac{\gamma}{R}+\frac{c_{1} k T}{\dot{X}_{0} \Omega_{d}} \frac{I_{0}(R / \rho)}{\rho}+c_{1} \frac{4 \sigma_{0}}{9 \dot{\varepsilon}_{0} \rho}\left[I_{0}(R / \rho)-I_{0}(r / \rho)\right],
$$

$$
\begin{gathered}
\sigma_{r r}=c_{1} \frac{2 \sigma_{0}}{3 \dot{\varepsilon}_{0}}\left(\frac{I_{1}(R / \rho)}{R}-\frac{I_{1}(r / \rho)}{r}\right)-\frac{\gamma}{R}, \\
\dot{\xi}_{n}=\frac{c_{1} I_{0}(r / \rho)}{\rho} .
\end{gathered}
$$

Subtracting Eq. (A11) from Eq. (A12) and setting $r=R$, we get

$$
\sigma_{r r}-p=\frac{k T}{\dot{X}_{0} \Omega_{d}} \frac{c_{1} I_{0}(R / \rho)}{\rho} \quad \text { at } \quad r=R .
$$

Substituting Eqs. (A10), (A13), and (A14) in Eq. (A2a), we find the solution for $c_{1}$,

$$
\begin{aligned}
c_{1}= & \frac{3 \dot{\varepsilon}_{z z} R}{2} \quad \frac{1}{(2+\Pi) I_{0}(R / \rho) R / \rho-3 I_{1}(R / \rho)}, \\
& \text { with } \Pi=\frac{9 \dot{\varepsilon}_{0} k T}{2 \sigma_{0} \Omega_{d} \dot{X}_{0}} .
\end{aligned}
$$

Equation (11) and the rest follow by substituting $c_{1}$ into Eqs. (A10)-(A13). The other stress components can be obtained by inverting Eqs. (A2b) and (A2c) and using the solutions from Eqs. (A10)-(A13).

Derivation of Eq. (16). In the limit $R / \rho \rightarrow 0$, viscous creep can be neglected. At steady state, Eq. (1) gives 


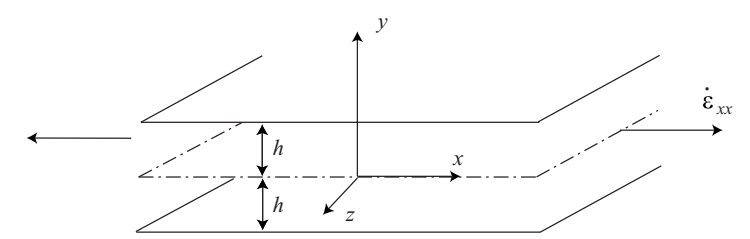

FIG. 15. The coordinate used for the deformation of a thin sheet (thickness $2 h$ ) under uniaxial tension.

$$
\dot{\varepsilon}_{r r}=\dot{\varepsilon}_{\theta \theta}=\dot{\varepsilon}_{z z}=\frac{\dot{\xi}_{n}}{3} .
$$

Now the chemical-potential function Eq. (5) can be written as

$$
\mu=\Omega_{d} p-3 \frac{k T}{\dot{X}_{0}} \dot{\varepsilon}_{z z} .
$$

From Eq. (7), at steady state, $\partial \xi / \partial t=0$, which leads to

$$
\frac{1}{r} \frac{\partial}{\partial r}\left(r \frac{D}{k T} \frac{\partial \mu_{v}}{\partial r}\right)+\dot{\xi}_{n}=0 .
$$

Using the mechanical equilibrium conditions, the boundary conditions, together with the above equation, we obtain Eq. (16).

\section{APPENDIX B}

In this appendix, we give a derivation of the analytical model of deformation in a thin plate or membrane by coupled diffusion and viscous flow, as shown in Fig. 15.

With Eqs. (1) and (2), we have

$$
\begin{aligned}
& \dot{\varepsilon}_{x x}=\frac{3 \dot{\varepsilon}_{0}}{2 \sigma_{0}}\left(\sigma_{x x}-p\right)+\frac{\dot{\xi}_{n}}{3}, \\
& \dot{\varepsilon}_{y y}=\frac{3 \dot{\varepsilon}_{0}}{2 \sigma_{0}}\left(\sigma_{y y}-p\right)+\frac{\dot{\xi}_{n}}{3}, \\
& \dot{\varepsilon}_{z z}=\frac{3 \dot{\varepsilon}_{0}}{2 \sigma_{0}}\left(\sigma_{z z}-p\right)+\frac{\dot{\xi}_{n}}{3} .
\end{aligned}
$$

Using the plane strain condition along the $z$ axis, we have $\dot{\varepsilon}_{z z}=0$, which gives

$$
p=\frac{\sigma_{x x}+\sigma_{y y}}{2}-\frac{\sigma_{0}}{9 \dot{\varepsilon}_{0}} \dot{\xi}_{n} .
$$

Since the plate is subjected to uniaxial tension, $\sigma_{x y}=0$ and we have $\sigma_{x z}=\sigma_{y z}=0$. The equations of equilibrium

$$
\frac{\partial \sigma_{x x}}{\partial x}+\frac{\partial \sigma_{x y}}{\partial y}+\frac{\partial \sigma_{x z}}{\partial z}=0
$$

$$
\frac{\partial \sigma_{x y}}{\partial x}+\frac{\partial \sigma_{y y}}{\partial y}+\frac{\partial \sigma_{x z}}{\partial z}=0,
$$

will be simplified to

$$
\frac{\partial \sigma_{x x}}{\partial x}=0, \quad \frac{\partial \sigma_{y y}}{\partial y}=0 .
$$

The solution of the stresses can be given as

$$
\sigma_{x x}=g(y), \quad \sigma_{y y}=f(x)=0 .
$$

To obtain the second term in Eq. (B5), we have used the stress boundary condition on the sample surface. Combining Eqs. (B1a), (B2), and (B5), the pressure can be rewritten as

$$
p=\frac{2 \sigma_{0}}{3 \dot{\varepsilon}_{0}}\left(\dot{\varepsilon}_{x x}-\frac{2}{3} \dot{\xi}_{n}\right)
$$

Substituting Eq. (B6) into the chemical potential Eq. (A5) and at steady state, we have

$$
\frac{D \Omega_{d}}{k T} \frac{\partial^{2}}{\partial y^{2}}\left[\frac{2 \sigma_{0}}{3 \dot{\varepsilon}_{0}}\left(\dot{\varepsilon}_{x x}-\frac{2}{3} \dot{\xi}_{n}\right)-\frac{k T}{\dot{X}_{0}} \dot{\xi}_{n}\right]+\dot{\xi}_{n}=0 .
$$

Using the definition for $\rho$ (Sec. III), the above equation has a general solution of

$$
\dot{\xi}_{n}=A e^{y / \rho}+B e^{-y / \rho}
$$

It is noted that by symmetry, the free-volume flux vanishes $(j=0)$ at $y=0$, leading to $A=B$ and $\dot{\xi}_{n}=2 A \cosh (y / \rho)$. The pressure is now given as

$$
p=\frac{2 \sigma_{0} \dot{\varepsilon}_{x x}}{3 \dot{\varepsilon}_{0}}-\frac{8 \sigma_{0}}{9 \dot{\varepsilon}_{0}} A \cosh (y / \rho) .
$$

Again, we regard the surface as a perfect sink for free volume, so that the excess free-volume concentration at a given temperature must remain at its equilibrium value $\xi_{s e}$ at the surface. With Eq. (3), we have

$$
k T \ln \left(\xi_{s e} / \xi_{e}\right)=\Omega_{d}\left[\frac{2 \sigma_{0} \dot{\varepsilon}_{x x}}{3 \dot{\varepsilon}_{0}}-\frac{8 \sigma_{0}}{9 \dot{\varepsilon}_{0}} A \cosh (h / \rho)\right] .
$$

To ensure Gibbs-Thompson condition at the surface, Eq. (9) gives $\xi_{s e}=\xi_{e}$ since the curvature of a flat surface is infinite. Hence we obtain

$$
\begin{aligned}
& A=\frac{1}{\cosh (h / \rho)} \frac{3 \dot{\varepsilon}_{x x}}{4}, \\
& \dot{\xi}_{n}=\frac{3 \dot{\varepsilon}_{x x}}{2} \frac{\cosh (y / \rho)}{\cosh (h / \rho)},
\end{aligned}
$$

and

$$
\sigma_{x x}=\frac{\sigma_{0} \dot{\varepsilon}_{x x}}{\dot{\varepsilon}_{0}}\left\{\frac{4}{3}-\frac{\cosh (y / \rho)}{\cosh (h / \rho)}\right\} .
$$


*Corresponding author. Present address: State Key Laboratory of Nonlinear Mechanics, Chinese Academy of Sciences, Beijing 100190, P.R. China. yujie_wei@1nm.imech.ac.cn

†allan_bower@brown.edu

*huajian_gao@brown.edu

${ }^{1}$ H. Guo, P. F. Yan, Y. B. Wang, J. Tan, Z. F. Zhang, M. L. Sui, and E. Ma, Nature Mater. 6, 735 (2007).

${ }^{2}$ Z. W. Shan, J. Li, Y. Q. Cheng, A. M. Minor, S. A. Syed Asif, O. L. Warren, and E. Ma, Phys. Rev. B 77, 155419 (2008).

${ }^{3}$ C. A. Volkert, A. Donohue, and F. Spaepen, J. Appl. Phys. 103, 083539 (2008).

${ }^{4}$ Y. Saotome and H. Iwazaki, J. Mater. Process. Technol. 119, 307 (2001).

${ }^{5}$ Z. Han, W. F. Wu, Y. Li, Y. J. Wei, and H. J. Gao, Acta Mater. 57, 1367 (2009).

${ }^{6}$ A. S. Argon, Acta Metall. 27, 47 (1979).

${ }^{7}$ P. A. Duine, J. Sietsma, and A. van den Beukel, Phys. Rev. B 48, 6957 (1993)

${ }^{8}$ U. Geyer, S. Schneider, W. L. Johnson, Y. Qiu, T. A. Tombrello, and M. P. Macht, Phys. Rev. Lett. 75, 2364 (1995).

${ }^{9}$ V. Zollmer, K. Ratzke, F. Faupel, A. Rehmet, and U. Geyer, Phys. Rev. B 65, 220201(R) (2002).

${ }^{10}$ F. Faupel, W. Frank, M. P. Macht, H. Mehrer, V. Naundorf, K. Ratzke, H. Schober, S. K. Sharma, and H. Teichler, Rev. Mod. Phys. 75, 237 (2003).

${ }^{11}$ C. Gaukel, M. Kluge, and H. R. Schober, Philos. Mag. B 79, 1907 (1999).

${ }^{12}$ Q. K. Li and M. Li, J. Non-Cryst. Solids 354, 2060 (2008).

${ }^{13}$ F. Delogu, Phys. Rev. Lett. 100, 255901 (2008).

${ }^{14}$ W. H. Wang, J. J. Lewandowski, and A. J. Greer, J. Mater. Res. 20, 2307 (2005).
${ }^{15}$ J. Das, M. B. Tang, K. B. Kim, R. Theissmann, F. Baier, W. H. Wang, and J. Eckert, Phys. Rev. Lett. 94, 205501 (2005).

${ }^{16}$ S. Kobayashi, K. Maeda, and S. Takeuchi, Acta Metall. 28, 1641 (1980).

${ }^{17}$ C. A. Schuh and A. C. Lund, Nature Mater. 2, 449 (2003).

${ }^{18}$ A. Păduraru, A. Kenoufi, N. P. Bailey, and J. Schiøtz, Adv. Eng. Mater. 9, 505 (2007).

${ }^{19}$ M. I. Mendelev, D. K. Rehbein, R. T. Ott, M. J. Kramer, and D. J. Sordelet, J. Appl. Phys. 102, 093518 (2007).

${ }^{20}$ S. J. Plimpton, J. Comput. Phys. 117, 1 (1995); http:// lammps.sandia.gov

${ }^{21}$ Y. Shi and M. L. Falk, Acta Mater. 55, 4317 (2007).

${ }^{22}$ F. Delogu, Phys. Rev. B 79, 184109 (2009).

${ }^{23}$ R. Huang, Z. G. Suo, J. H. Prevost, and W. D. Nix, J. Mech. Phys. Solids 50, 1011 (2002).

${ }^{24}$ D. B. Miracle, Nature Mater. 3, 697 (2004).

${ }^{25}$ H. W. Sheng, H. Z. Liu, Y. G. Cheng, J. Wen, P. L. Lee, W. K. Luo, S. D. Shastri, and E. Ma, Nature Mater. 6, 192 (2007).

${ }^{26}$ F. Spaepen, Acta Metall. 25, 407 (1977).

${ }^{27}$ Q. Yang, A. Mota, and M. Ortiz, Comput. Mech. 37, 194 (2006).

${ }^{28}$ P. S. Steif, F. Spaepen, and J. W. Hutchinson, Acta Metall. 30, 447 (1982).

${ }^{29}$ K. M. Flores and R. H. Dauskardt, Acta Mater. 49, 2527 (2001).

${ }^{30}$ S. V. Nemilov, Glass Phys. Chem. 21, 379 (1995).

${ }^{31}$ C. A. Angell, Science 267, 1924 (1995).

${ }^{32}$ T. A. Waniuk, R. Busch, A. Masuhr, and W. L. Johnson, Acta Metall. 46, 5229 (1998).

${ }^{33}$ J. Lu, G. Ravichandran, and W. L. Johnson, Acta Mater. 51, 3429 (2003).

${ }^{34}$ K. Ohsaka, S. K. Chung, W. K. Rhim, A. Peker, D. Scruggs, and W. L. Johnson, Appl. Phys. Lett. 70, 726 (1997). 